\title{
Investigation of the Size Effect in Euler-Bernoulli Nanobeam Using the Modified Couple Stress Theory
}

\author{
Necla Togun ${ }^{1, *}$, Süleyman Murat Bağdatl1 ${ }^{2}$ \\ ${ }^{1}$ Vocational School of Technical Sciences in Gaziantep, University of Gaziantep, 27310, Gaziantep, Turkey, \\ Phone: +90 342 3171791, nkara@gantep.edu.tr \\ ${ }^{2}$ Faculty of Engineering, Department of Mechanical Engineering, Celal Bayar University, 45140, Yunusemre, Manisa, Turkey, \\ Phone: +90 236 2012368, murat.bagdatli@.cbu.edu.tr \\ ${ }^{*}$ Corresponding Author
}

Received: 3 March 2017

Accepted: 6 November 2017

DOI: $10.18466 /$ cbayarfbe. 370362

\begin{abstract}
This paper presents the implementation of non-classical continuum theory for simply supported nanobeam. Hamilton's principle and modified couple stress methods are employed for obtaining differential equation of motion of nanobeam in cooperation with suitable boundary conditions. An approximate solution of the presented system is developed considering the method of multiple scales which is one of the perturbation techniques. The effect of material length scale parameter $\zeta$ and the Poisson's ratio $v$ on the natural frequencies are determined and represented in table form and graphically. Besides, dimensionless natural of frequency of nanobeam are investigated by taking into account various system parameters. The results of the system show that the size influence is very crucial for extremely thin beams with a height of nanoscale dimension. Besides, the outcome of the system shows that the beam modeled considering non-classical continuum theory is stiffer than those of classical one.
\end{abstract}

Keywords: Modified couple stress theory, nanobeam, perturbation method, vibration

\section{Introduction}

The ultrahigh frequency mixers and ultrahigh precision sensors can be exactly manufactured in current nanotechnology. The mechanical properties of micro/nano-electromechanical system (MEMS/NEMS) based devices like resonators, actuators, sensors and mixers are necessary to be well characterized in the purpose of understanding the principle of these nanostructures. Micro/nano-beam subject that was the most common components in MEMS/NEMS has been attracted the attention of researchers in recent years [1-3].

In recent years, studies related to nanostructure, which are extensively used as the principle part of numerous MEMS and NEMS, have received great attention among scientists. Classical MEMS and NEMS make up of metal extreme thin beams, conventional silicon-based materials, polymers, or functionally graded materials. The mechanical and dynamic behavior of nanostructures has attracted particular attention from researchers. The approach to developing non-classical continuum theories for modeling static and dynamic movement of micro and nanostructures is im- portant because it is difficult and time consuming to conduct experiments at the nanoscale. With non-classical continuum theories to analyze nanostructures, it is possible to introduce material length parameters in the principle relations to define the size influence.

Various size dependent continuum theories is more appealing research topic for engineers and scientist, and with the introduction of size effect in the constitutive relations, linear and nonlinear mechanical characteristics of nanostructures have been captured. Those of which includes the nonlocal elasticity theory [4], the modified couple stress theory [5], the surface elasticity [6], the strain gradient theory [7] and the micropolar theory [8]. Park and Gao [9] initially developed the formulation of EulerBernoulli beam theory using modified couple stress theory (MCST) due to analyze the static deformation of a micro cantilever beam subjected to a point load.

Recently, scientists have been attempting to use the couple stress effect due to capturing size effects of nanostructures. In the literature, several beam models taking into account 
Celal Bayar University Journal Of Science

Volume 13, Issue 4, p 893-899

of the Timoshenko, Euler-Bernoulli and Reddy beam theories have been proposed in order to research the influence of the size effect on nanobeam models for analyzing the mechanical properties. Kong et al. [10] studied the dynamic model for Euler-Bernoulli beams considering MCST. They assessed the fundamental frequencies of the beams with size-dependeny. They found that the fundamental frequencies determined by the newly established model decreases with increase of the typical sizes ratio to interor material length scale parameter. Şimşek [11] presented dynamic response of an embedded Euler-Bernoulli type microbeam considering the effect of moving microparticle on the base of MCST. A study presented by Şimşek [11] found that dynamic analysis are generally beneficial for the accurate design of systems like as sensors, actuators, atomic force microscopes and microtubes conveying microparticles and of microscale devices. Asghari et al. [12-15] studied the size effects in Timoshenko beam model account of the MCST. Their results indicated that modeling by in that theory has more robust than modeling by the classical beam theory. Fu and Zhang [16] presented a study deal with the effect of size parameter in Timoshenko beam model using MCST for the purpose of investigating the mechanical behaviors of microtubes. Their result shows that the more increase the amount of material length scale parameter, the more the buckling wave quantity minimizes correspondingly as soon as it disappears and also the more decrease the regarding buckling amplitude. The free vibration and static bending subject of a simply supported beam were studied by nonclassical beam model [17]. Their result reveals that the fundamental frequency obtained by the proposed model is greater than those by the classical model. And also, the distinction between them becomes considerably major just for extreme thin beams. Chen et al. [18] used MCST to the newly developed model of laminated Reddy beam. Their numerical result reveals that the proposed beam model finds out the microstructure scale-effect. They can also observe that the stresses and deflections carried out by the proposed model are smaller than those based on Timoshenko and Euler-Bernoulli beam assumptions. These studies were pursued in [19-23] who studied the effect of size parameter on the beam model considering MCST. Kocatürk and Akbaş [24] presented responses of the free end of a cantilever micro beam under the effect of an impact force considering the MCST. Their numerical results reveal that the classical beam theory based on the MCST must be used instead of the classical theory for small values of beam height. Akbaş [25] investigated the forced vibration of viscoelastic nanobeams embedded in an elastic medium and showed that with increase in the aspect ratio and the nondimensional parameter of material length scale, the difference between the dynamic responses of classical beam theory and MCST decrease considerably. Another study of Akbaş [26] dealed with the static bending of edge fractured
N. Togun

micro beams under uniformly distributed transverse loading based on MCST. Civalek [27] studied to analyze the free vibration of cylindrical and conical shells and annular plates made of composite laminated and functionally graded materials (FGMs). Shen and Li [28] used semi-continuum model to investigate the bending charactersitics of very thin micro/nano-beams. They showed that their obtained results might be helpful in designing and understanding the very thin micro/nano-beams by considering MEMS/NEMS. Semmani et. al [29] presented a study related to free bending vibration behavior of geometrically defective functionally graded (FG) microbeams. They used MCST due to capturing size effect.

The literature related to nonlinear models is rather limited compared to the linear ones. The influence of various system parameters on the nonlinear resonant dynamics of a microscale beam were studied by some researchers to investigate the nonlinear forced dynamics of the system via MCST [30] and strain gradient theory [31]. Wang et al [32] presented a study associated with the nonlinear free vibration of the microbeams by using MCST. They concluded that the nonlinear vibration of a microbeam has a hardening spring behavior, and beam models related to MCST are stiffer than those based on the classical beam theory. Xia et al. [33] used the MCST for the purpose of nonlinear analysis of microbeams and investigated the postbuckling, static bending and free vibration by taking into account the material length scale parameter. Şimşek [34] carried out the static and nonlinear vibration analysis of microbeams embedded in nonlinear elastic foundation by using non-classical beam theory. His numerical results show that the nonlinear frequency ratio would decrease as the dimensionless scale parameter magnifies, and the nonlinear foundation parameter has the reverse effect on the nonlinear frequency according to Winkler and the Pasternak types.

There are number of papers in the literature about nanobeams and microbeams. Many of these are focuses on nonlocal effects on the linear and nonlinear problems to analyze the dynamic characteristics. In this study, linear free vibration of a simply-supported nanobeam is analyzed numerically by using MCST. The closed form solution is dependent on the multiple scale method and the approximate solution by using the Hamilton's equations is determined. A comprehensive parametric study is performed to analyze the effects of the material length scale parameter and the Poisson's ratio. Frequencies related to natural based on classical beam theory and modified couple stress theory are obtained and checked against previous studies in order to show a relation with each other.

\section{The modified couple stress theory}

The classical couple stress theory was firstly presented for 
Celal Bayar University Journal Of Science

Volume 13, Issue 4, p 893-899

isotropic elastic materials in 1960s by Toupin [35], Mindlin and Tiersten [36], Mindlin [37]. This theory is composed of two higher order material length scale parameters and Lame constants to include the size effects. It is noted that this theory has the smallest material length scale parameters than strain gradient and nonlocal theory. The idea that the MCST was initially pioneered by Yang et al. [5] to improve the accuracy of the studies performing in the area of nanostructure. This theory mentions that the density of strain energy is a function of not only strain tensor but also curvature tensor. Thus, the strain energy of a deformed isotropic linear elastic body occupying a volume $\Omega$ is given as

$$
U=\frac{1}{2} \int_{\Omega}\left(\sigma_{i j} \varepsilon_{i j}+m_{i j} \chi_{i j}\right) d V i, j=1,2,3
$$

in where $\sigma_{i j} i s$ the stress tensor, $\varepsilon_{i j}$ is the strain tensor, $m_{i j}$ is the deviatoric part of the couple stress tensor and $\chi_{i j}$ is the symmetric curvature tensor defined by

$$
\begin{gathered}
\sigma_{i j}=\lambda \varepsilon_{k k} \delta_{i j}+2 \mu \varepsilon_{i j} \\
\varepsilon_{i j}=\frac{1}{2}\left(u_{i, j}+u_{j, i}\right) \\
m_{i j}=2 \mu l^{2} \chi_{i j} \\
\chi_{i j}=\frac{1}{2}\left(\theta_{i, j}+\theta_{j, i}\right)
\end{gathered}
$$

where $u_{i}$ is the displacement vector, $\delta_{i j}$ is the Kronecker delta, $l$ is the material length scale parameter. $\theta_{i}$ is the rotation vector that can be defined as

$$
\theta_{i}=\frac{1}{2} e_{i j k} u_{k, j}
$$

where $e_{i j k}$ is the permutation symbol. $\lambda$ and $\mu$ are the Láme's constants defined as

$$
\lambda=\frac{\mathrm{E} v}{(1+v)(1-2 v)}, \quad \mu=\frac{\mathrm{E}}{2(1+v)}
$$

where $v$ is Poisson's ratio, $\mu$ is shear modulus and $E$ is modulus of elasticity.

\section{The equation of motion}

In the present study, Hamilton's principle and modified coupled stress theory are implemented in an existing EulerBernoulli beam with size dependent and simply supported beam is taken as a boundary condition shown in Figure 1. For the system shown in Figure 1, L is the beam length.

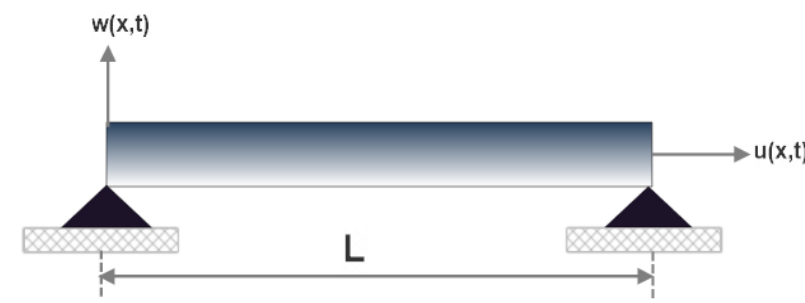

Figure 1. Simply supported beam

On the bases of the Euler-Bernoulli beam theory, the displacement field is given as

$$
u=-z \psi(x, t), \quad v=0, \quad w=w(x, t)
$$

where, $u, v$ and $w$ are the displacement components along $\mathrm{x}, \mathrm{y}, \mathrm{z}$ axes, respectively. $\psi$ is the rotation angle of sections related with deflections given as

$$
\psi(x, t)=\frac{\partial w(x, t)}{\partial x}
$$

The Poisson's effect can be disregarded and the principal parts of the stress and the couple stress tensors can be given by using Equation (2.2)-(2.6)

$$
\begin{aligned}
\varepsilon_{x x} & =-z \frac{\partial^{2} w(x, t)}{\partial x^{2}} \\
\theta_{y} & =-\frac{\partial w(x, t)}{\partial x} \\
\chi_{x y} & =-\frac{1}{2} \frac{\partial^{2} w(x, t)}{\partial x^{2}} \\
\sigma_{x x} & =-E z \frac{\partial^{2} w(x, t)}{\partial x^{2}} \\
m_{x y} & =-\mu l^{2} \frac{\partial^{2} w(x, t)}{\partial x^{2}}
\end{aligned}
$$

Potential (U) and kinetic (T) energy expressions of the system are written to obtain the equations of motion of size dependent nanobeam shown in Figure 1. The potential energy $U$ can be written by using Equations (2.1), (2.2), (2.4), (3.3) and (3.5)

$$
\begin{gathered}
U=\frac{1}{2} \int_{0}^{L}\left(\frac{E(1-v) I}{(1+v)(1-2 v)}+\mu A l^{2}\right)\left(\frac{\partial^{2} w}{\partial x^{2}}\right)^{2} d x+ \\
\frac{1}{2} \int_{0}^{L} N\left[\frac{\partial u}{\partial x}+\frac{1}{2}\left(\frac{\partial w}{\partial x}\right)^{2}\right] d x
\end{gathered}
$$

where, $I$ is the moment of inertia and A is the area of crosssection. The kinetic energy $(T)$ formula can be obtained as

$$
T=\frac{1}{2} \rho A \int_{0}^{L}\left[\left(\frac{\partial u}{\partial t}\right)^{2}+\left(\frac{\partial w}{\partial t}\right)^{2}\right] d x
$$

where, $\rho A$ is the mass per unit length. The Hamilton's principle can be written according to form below:

$$
\delta \int_{0}^{t}\left[T-\left(U-W_{\text {ext }}\right)\right] d t=0
$$

where $\delta W_{\text {ext }}=0$; inserting Equations (3.8) and (3.9) into 
Celal Bayar University Journal Of Science

Volume 13, Issue 4, p 893-899

Equation (3.10) and integrating by parts, and collecting the coefficients of $\delta w$, the following motion equations for the Euler-Bernoulli beam with size dependent considering the MCST and including the Poison influence are obtained

$$
\begin{gathered}
\left(\frac{E(1-v) I}{(1+v)(1-2 v)}+\mu A l^{2}\right) \frac{\partial^{4} w}{\partial x^{4}}+\rho A \frac{\partial^{2} w}{\partial t^{2}}= \\
\frac{E A}{2 L} \int_{0}^{L}\left[\left(\frac{\partial w}{\partial x}\right)^{2} d x\right] \frac{\partial^{2} w}{\partial x^{2}}
\end{gathered}
$$

Note that here when the length scale parameter $l$ and the Poisson's ratio $v$ are receive to be zero in Equation (3.11), the classical Euler-Bernoulli beam equation can be acquired. Effect of Poisson's will have to be inserting into equations because of accurate and reliable results [31]. The following dimensionless quantities are expressed because of the independent of geometrical properties and beam material as follows:

$$
\begin{aligned}
& \bar{x}=\frac{x}{L}, \bar{w}=\frac{w}{L}, \bar{t}=\frac{t}{L^{2}} \sqrt{\frac{E I}{\rho A}}, \\
& \xi=\frac{h}{l}, \quad \eta=\frac{6(1-2 v)}{(1-v) \xi^{2}} \\
& \alpha=\frac{(1-v)}{(1+v)(1-2 v)}
\end{aligned}
$$

The Equations(3.11) and (3.12) is integrated to obtain general dimensionless form of equation of motion as follows:

$$
(1+\eta) \frac{\partial^{4} \bar{w}}{\partial \bar{x}^{4}}+\frac{1}{\alpha} \frac{\partial^{2} \bar{w}}{\partial \bar{t}^{2}}=\frac{1}{2 \alpha}\left[\int_{0}^{1}\left(\frac{\partial \bar{w}}{\partial \bar{x}}\right)^{2} d \bar{x}\right] \frac{\partial^{2} \bar{w}}{\partial \bar{x}^{2}}
$$

The non-dimensional form of simply supported boundary condition at the beam end (at $\mathrm{x}=0$ and $\mathrm{x}=1$ ) can be written as

$$
\begin{array}{ll}
\bar{w}(0)=0, & \bar{w}(1)=0 \\
\bar{w}^{\prime \prime}(0)=0, & \bar{w}^{\prime \prime}(1)=0
\end{array}
$$

\section{Approximate Solutions}

Method of multiple scales, the perturbation techniques, is used to acquire the approximate solutions of the equation of motion [38, 39]. Slow time scale $T_{0}=t$ and fast time scale, $T_{l}=\varepsilon t$ can be written to present the orders. In slow and fast time scales derivatives are taken with respect to time as

$$
\begin{aligned}
& \partial / \partial t=D_{0}+\varepsilon D_{1} \text { and } \\
& \partial^{2} / \partial t^{2}=D_{0}{ }^{2}+2 \varepsilon D_{0} D_{1} \text { where } D_{n}=\partial / \partial T_{n}
\end{aligned}
$$

The solution of the nanobeam model is assumed as:
N. Togun

$$
\bar{w}(x, t: \varepsilon)=\bar{w}_{0}\left(x, T_{0}, T_{1}\right)+\varepsilon \bar{w}_{1}\left(x, T_{0}, T_{1}\right)
$$

Obtained equations are separated into the orders 1 and $\varepsilon$. Order 1 being linear part of the system and orders $\varepsilon$ being nonlinear part of the system are written by following formula:

Order (1):

$$
(1+\eta) \bar{w}_{0}^{i v}+\frac{1}{\alpha} D_{0}^{2} \bar{w}_{0}=0
$$

Order $(\varepsilon)$

$$
\begin{aligned}
& (1+\eta) \bar{w}_{1}^{i v}+\frac{1}{\alpha} D_{0}^{2} \bar{w}_{1}=-\frac{2}{\alpha} D_{0} D_{1} y_{0}+ \\
& \frac{1}{2 \alpha}\left[\int_{0}^{1} \bar{w}_{0}^{\prime \prime} 2 d x\right] \bar{w}_{0}^{\prime \prime}+F \cos \Omega \mathrm{t}-2 \mu D_{0} \bar{w}_{0}
\end{aligned}
$$

\subsection{Linear problem}

The order 1 given in Equation (3.16) constitutes the linear problem of the system. The complex form is written for the solution of the problem given as:

$\bar{w}_{0}\left(x, T_{0}, T_{1}\right)=A\left(T_{1}\right) e^{i \omega \mathrm{T}_{0}} Y(x)+\bar{A}\left(T_{1}\right) e^{-i \omega \mathrm{T}_{0}} \bar{Y}(x)$

in which $\mathrm{A}$ is the complex amplitude. Inserting Equation (4.1) into Equation (3.16) results in the following formula:

$$
(1+\eta) Y^{i v}(x)-\frac{\omega^{2}}{\alpha} Y(x)=0
$$

Solution of $\mathrm{Y}(\mathrm{x})$ can be in the following form:

$$
Y(x)=c_{1} e^{i \beta_{1} x}+c_{2} e^{i \beta_{2} x}+c_{3} e^{i \beta_{3} x}+c_{4} e^{i \beta_{4} x}
$$

The applied the boundary conditions, the obtained the frequency equations.

\section{Numerical results}

In this section, vibration characteristic of the simply supported nanobeam is analyzed by using MCST. The material length scale parameter $(l)$ is included into the equations to capture the size-effect. In view of Equation (19), $\xi$ which expresses as a function of $h / l$. It figures out that nanobeam sensitivity is a function of the ratio of the beam height to the internal material length scale parameter $h / l$ that reports the size dependent behavior of the nanobeam $[14,40]$. In Table 1 , the fundamental frequency of a nanobeam as a function of dimensionless material length scale parameter $\xi$ (i.e., the ratio of beam height to the material length scale parameter, $h / l$ ) and as a function of Poisson effect and without the Poison effect are considered. It is obvious from Table 1 that frequency of the 
beam is dependent on the $\xi$ value and besides the linear frequency of the non-classical beam theory approaches to classical beam theory until the dimensionless parameter $\xi$ increases further. The fundamental frequency acquired by considering MCST is always greater than those by classical beam theory because of the rising bending rigidity investigated by the non-classical Euler-Bernoulli beam model. In addition, the results of the system indicate that the beam modeled taking into account of MCST is stiffer than those of classical one. It is noted that, Poisson's ratio inflence decreases with the value of $\xi$ increasing. It is notable that natural frequencies acquired by the MCST based on different quantities of $\xi$ and $v$ is greater than those acquired by the classical beam theory. For a given $\xi$, the fundamental frequency related with the Poisson's ratio influence is greater than those without Poisson's ratio effect.

Figure 2 presents the relation between the dimensionless natural frequency and the material length scale parameter for different values of the Poison's ratio. Four different Poisson's ratio (i.e., $v=0,0.23,0.38$ and 0.45 ) are considered. Figure 2 distinctly presents that the natural frequencies are nonlinearly dependent on the material length scale parameter $(\zeta)$ and the natural frequencies decrease with the increase in the value of $\zeta$. However, the difference between the results becomes more unclear for high ratio of height to length scale $\mathrm{h} / \mathrm{l}$. Furthermore, the effect of $\mathrm{h} / \mathrm{l}$ is decreased or even diminished with the increase in the ratio. This is due to the value of material length scale parameter is increased, beam becomes stiffer. Influence of size on the value of fundamental frequency is obviously seen only when the height of the beam is extremely little (with $h / l<4$ ). This implies one more that influence of size has a significant influence on extremely thin beams means that height is at the nanoscale.

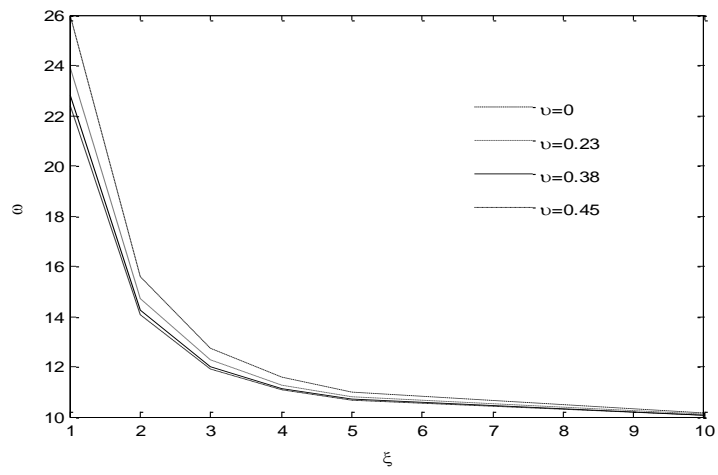

Figure 2. Effect of Poisson on the natural frequency of the simply supported nanobeam

Table 1. Non-dimensional natural frequencies of a beam with simply supported.

\begin{tabular}{|c|c|c|c|c|c|c|c|}
\hline \multirow{3}{*}{$v$} & \multirow{3}{*}{$\xi$} & \multicolumn{6}{|c|}{ Non-dimensional natural frequencies } \\
\hline & & \multicolumn{3}{|c|}{ Without Poisson effect } & \multicolumn{3}{|c|}{ With Poisson effect } \\
\hline & & $\omega_{1}$ & $\omega_{2}$ & $\omega_{3}$ & $\omega_{1}$ & $\omega_{2}$ & $\omega_{3}$ \\
\hline \multirow{7}{*}{0.0} & 1.0 & 26.1125 & 104.45 & 235.013 & 26.1125 & 104.45 & 235.013 \\
\hline & 2.0 & 15.6052 & 62.4209 & 140.447 & 15.6052 & 62.4209 & 140.447 \\
\hline & 3.0 & 12.7416 & 50.9664 & 114.674 & 12.7416 & 50.9664 & 114.674 \\
\hline & 4.0 & 11.5731 & 46.2925 & 104.158 & 11.5731 & 46.2925 & 104.158 \\
\hline & 5.0 & 10.9903 & 43.9613 & 98.9129 & 10.9903 & 43.9613 & 98.9129 \\
\hline & 10.0 & 10.1614 & 40.6455 & 91.4524 & 10.1614 & 40.6455 & 91.4524 \\
\hline & Classical beam & 9.8696 & 39.4784 & 88.8264 & 9.8696 & 39.4784 & 88.8264 \\
\hline \multirow{7}{*}{0.23} & 1.0 & 23.9285 & 95.7142 & 215.357 & 24.2506 & 97.0024 & 218.255 \\
\hline & 2.0 & 14.7038 & 58.8151 & 132.334 & 15.2223 & 60.889 & 137.000 \\
\hline & 3.0 & 12.2558 & 49.0233 & 110.302 & 12.8733 & 51.4932 & 115.86 \\
\hline & 4.0 & 11.2742 & 45.0967 & 101.468 & 11.9425 & 47.77 & 107.482 \\
\hline & 5.0 & 10.7896 & 43.1585 & 97.1065 & 11.4862 & 45.9447 & 103.375 \\
\hline & 10.0 & 10.1075 & 40.4298 & 90.9671 & 10.8479 & 43.3916 & 97.6311 \\
\hline & Classical beam & 9.8696 & 39.4784 & 88.8264 & 10.6266 & 42.5065 & 95.6397 \\
\hline \multirow{7}{*}{0.38} & 1.0 & 22.8238 & 91.2953 & 205.414 & 24.6143 & 98.4574 & 221.529 \\
\hline & 2.0 & 14.2579 & 57.0317 & 128.321 & 16.9773 & 67.909 & 152.795 \\
\hline & 3.0 & 12.0194 & 48.0777 & 108.175 & 15.1461 & 60.5845 & 136.315 \\
\hline & 4.0 & 11.1301 & 44.5204 & 100.171 & 14.4505 & 57.8021 & 130.055 \\
\hline & 5.0 & 10.6934 & 42.7738 & 96.241 & 14.1170 & 56.4679 & 127.053 \\
\hline & 10.0 & 10.0819 & 40.3275 & 90.7369 & 13.6595 & 54.6382 & 122.936 \\
\hline & Classical beam & 9.8696 & 39.4784 & 88.8264 & 13.5036 & 54.0145 & 121.533 \\
\hline
\end{tabular}


Celal Bayar University Journal Of Science

Volume 13, Issue 4, p 893-899

N. Togun

\begin{tabular}{cccccccc}
\hline & 1.0 & 22.3714 & 89.4857 & 201.343 & 27.7949 & 111.179 & 250.154 \\
& 2.0 & 14.0775 & 56.3102 & 126.698 & 21.6853 & 86.7411 & 195.167 \\
0.45 & 3.0 & 11.9245 & 47.6982 & 107.321 & 20.3536 & 81.4144 & 183.182 \\
& 4.0 & 11.0725 & 44.2902 & 99.6528 & 19.8664 & 79.4657 & 178.798 \\
& 5.0 & 10.6551 & 42.6206 & 95.8962 & 19.6368 & 78.5474 & 176.732 \\
& 10.0 & 10.0717 & 40.2869 & 90.6456 & 19.3265 & 77.306 & 173.938 \\
& Classical beam & 9.8696 & 39.4784 & 88.8264 & 19.2219 & 76.8877 & 172.997 \\
\hline
\end{tabular}

\section{Conclusions}

This paper presented the free vibration of nanobeams with simply supported by using Euler-Bernoulli beam theory and the MCST. The nonlinear equation of motion and boundary condition are derived by using the Hamilton's principle. In the present paper, method of multiple scales is implemented to acquire the approximate solutions of the motion eqautions. The influences of the material length scale parameter $(\zeta)$ and the Poison's ratio $(v)$ on the natural frequencies are investigated. Also, the considerable amount of numerical data is listed in tabular form for different quantities of the parameters in order to be references for future studies. In brief, numerical result show that the nondimensional natural frequency would decrease as the dimensionless scale parameters magnifies.

\section{References}

1. Flannigan, D.J.; Samartzis, P.C.; Yurtsever, A.; Zewail, A.H. Nanomechanical motion of cantilevers: direct imaging in real space and time with 4D electron microscopy, Nano Letters, 2009, 9(2), 875-81.

2. Beni, Y.T.; Karimipour, I.; Abadyan, M. Modeling the instability of electrostatic nano-bridges and nano-cantilevers using modified strain gradient theory, Applied Mathematical Modelling, 2015, 39(9), 2633 2648.

3. Korayem, A.H.; Kianfar, A.; Korayemn, M.H. Modeling and simulating of V-shaped piezoelectric micro-cantilevers using MCS theory considering the various surface geometries, Physica E, 2016, 84, 268279.

4. Eringen, A.C. Nonlocal polar elastic continua, International Journal of Engineering Science, 1972, 10, 1-16.

5. Yang, F.; Chong, A.C.M.; Lam, D.C.C.; Tong, P. Couple stress based strain gradient theory for elasticity, International Journal of Solids and Structures, 2002, 39, 2731-2743.

6. Gurtin, M.E.; Weissmuller, J.; Larche, F. A general theory of curved deformable interfaces in solids at equilibrium, Philosophical Magazine $A, 1998,78(5), 1093-1109$.

7. Aifantis, E.C. Strain gradient interpretation of size effects, International Journal of Fracture, 1999, 95, 1-4.

8. Eringen, A.C. Theory of micropolar plates, Zeitschrift fur Angewandte Mathematik und Physik, 1967, 18, 12-30.

9. Park, S.K.; Gao, X.L. Bernoulli-Euler beam model based on a modified couple stress theory, Journal of Micromechanics and Microengineering, 2006, 16, 2355-2359.

10. Kong, S,; Zhou, S.; Nie, Z.; Wang, K. The size-dependent natural fre- quency of Bernoulli-Euler micro-beams, International Journal of Engineering Science, 2008, 46, 427-437.

11. Şimşek, M. Dynamic analysis of an embedded microbeam carrying a moving microparticle based on the modified couple stress theory, International Journal of Engineering Science, 2010, 48, 1721-1732.

12. Asghari, M,; Kahrobaiyan, M.H.; Ahmadian, M.T. A nonlinear Timoshenko beam formulation based on the modified couple stress theory, International Journal of Engineering Science, 2010, 48, 1749-1761.

13. Asghari, M.; Kahrobaiyan, M.H.; Rahaeifard, M.; Ahmadian, M.T. Investigation of the size effects in Timoshenko beams based on the couple stress theory, Archive of Applied Mechanics, 2011, 81, 863-874.

14. Kahrobaiyan, M.H.; Asghari, M.; Ahmadian, M.T. A Timoshenko beam element based on the modified couple stress theory, International Journal of Mechanical Science, 2014, 79, 75-83.

15. Kahrobaiyan, M.H.; Asghari, M.; Rahaeifard, M.; Ahmadian, M.T. Investigation of the size-dependent dynamic characteristics of atomic force microscope microcantilevers based on the modified couple stress theory, International Journal of Engineering Science, 2010, 48, 1985-1994.

16. Fu, Y.; Zhang, J. Modeling and analysis of microtubules based on a modified couple stress theory, Physica E, 2010, 42, 1741-1745.

17. Ma, H.M.; Gao, X.L.; Reddy, J.N. A microstructure-dependent Timoshenko beam model based on a modified couple stress theory, Journal of Mechanics and Physics of Solids, 2008, 56, 3379-3391.

18. Chen, W.; Weiwei, C.; Sze, K.Y. A model of composite laminated Reddy beam based on a modified couple-stress theory, Composite Structures, 2012, 94, 2599-2609.

19. Ke, L.L.; Wang, Y.S. Flow-induced vibration and instability of embedded double-walled carbon nanotubes based on a modified couple stress theory, Physica E, 2011, 43, 1031-1039.

20. Roque, C.M.C.; Fidalgo, D.S.; Ferreira, A.J.M.; Reddy, J.N. A study of a microstructure-dependent composite laminated Timoshenko beam using a modified couple stress theory and a meshless method, Composite Structures, 2013, 96, 532-537.

21. Baghani, M. Analytical study on size-dependent static pull-in voltage of microcantilevers using the modified couple stress theory, International Journal of Engineering Science, 2012, 54, 99-105.

22. Togun, N.; Bağdatlı, S.M. Size dependent nonlinear vibration of the tensioned nanobeam based on the modified couple stress theory, Composites Part B: Engineering, 2016, 97, 255-262.

23. Atc1, D.; Bağdatlı, S.M. Vibrations of fluid conveying microbeams under non-ideal boundary conditions, Microsystem Technologies, 2017, 1-12.

24. Kocaturk, T.; Akbas, S.D. Wave propagation in a microbeam based on 
the modified couple stress theory, Structural Engineering and $\mathrm{Me}$ chanics, 2013, 46(3), 417-431.

25. Akbas, S.D. Forced vibration analysis of viscoelastic nanobeams embedded in an elastic medium, Smart Structures and Systems, 2016, 18(6), 1125-1143.

26. Akbas, S.D. Analytical solutions for static bending of edge cracked micro beams, Structural Engineering and Mechanics, 2016, 59(3), 579-599.

27. Civalek, Ö. Free vibration of carbon nanotubes reinforced (CNTR) and functionally graded shells and plates based on FSDT via discrete singular convolution method, Composites Part B: Engineering, 2017, $111,45-59$.

28. Shen, J.P.; Li, C. A. Semi-continuum-based bending analysis for extreme-thin micro/nano-beams and new proposal for nonlocal differential constitution, Composite Structures, 2017, 172, 210-220.

29. Dehrouyeh-Semnani, A.M.; Mostafaei, H.; and Nikkhah-Bahrami, M. Free flexural vibration of geometrically imperfect functionally graded microbeams, International Journal of Engineering Science, 2016, 105, 56-79.

30. Ghayesh, M.H.; Farokhi, H.; Amabili, M. Nonlinear dynamics of a microscale beam based on the modified couple stress theory, Composites: Part B, 2013a, 50, 318-324.

31. Ghayesh, M.H.; Amabili, M.; Farokhi, H. Nonlinear forced vibrations of a microbeam based on the strain gradient elasticity theory, International Journal of Engineering Science, 2013b, 63, 52-60.
32. Wang, Y.G.; Lin, W.H.; Liu, N. Nonlinear free vibration of a microscale beam based on modified couple stress theory, Physica E, 2013, 47, 80-85.

33. Xia, W.; Wang, L.; Yin, L. Nonlinear non-classical microscale beams: Static bending, postbuckling and free vibration, International Journal of Engineering Science, 2010, 48, 2044-2053.

34. Şimşek, M. Nonlinear static and free vibration analysis of microbeams based on the nonlinear elastic foundation using modified couple stress theory and He's variational method, Composite Structures, 2014, 112, 264-272.

35. Toupin, R.A. Theories of elasticity with couple stress, Archive for Rational Mechanics and Analysis, 1962, 17, 85-112.

36. Mindlin, R.D.; Tiersten, H.F. Effects of couple-stresses in linear elasticity, Archive for Rational Mechanics and Analysis, 1962, 11, 415448.

37. Mindlin, R.D., Influence of couple-stresses on stress concentrations, Experimental Mechanics, 1964, 3, 1-7.

38. Nayfeh, A.H., Mook, D.T. Nonlinear Oscillations; John Wiley, New York, 1979.

39. Nayfeh, A.H. Introduction to Perturbation Techniques, John Wiley, New York, 1981.

40. Barretta, R.; Luciano, R.; Willis, J.R. On torsion of random composite beams, Composite Structures, 2015, 132, 915-922. 